\title{
El paralelismo entre el evento de ayer y el de hoy: exhibiciones de poder
}

\author{
Magdalena Mut CAMACHO \\ Universitat Jaume I de Castellón \\ magda.mut@uji,es \\ Estela BERnAD MONFERRER \\ Universitat Jaume I de Castellón \\ bernad@uji.es
}

\begin{abstract}
Resumen:
El evento de empresa de hoy en día es una técnica de comunicación donde el objetivo último es la conexión emocional entre la empresa y el público. Haciendo un análisis abstracto del evento, éste es una experiencia global donde todo comunica, pensada en función de los intereses de quien lo promueve, donde la comunicación cara a cara alcanza su máximo exponencial, para que llegue un mensaje determinado a los que participan en el acto. Los eventos tienen el poder de comunicar a través de sensaciones, pues son espacios donde la información se vive, se siente y se toca. Por todo ello, se puede derivar que los eventos son herramientas que eficientemente utilizadas y con creatividad, tienen poder y son, en sí mismos, una demostración de poder.

Esta técnica de comunicación, con ese objetivo de relación, no es nueva. Si recurrimos a la historia encontramos una época pasada en la que la forma de comunicar poder y riqueza eran los eventos y quien los hacían eran los reyes.

$\mathrm{Si}$ indagamos en la memoria ya localizamos en las intenciones de buena parte de las actividades comunicativas de los estamentos públicos de épocas pretéritas, sobre todo los monarcas, una clara intención de persuadir y de incidir, a través de las acciones de comunicación, en la conformación de una imagen positiva de la monarquía.

Hoy en día, es la empresa la que ha recogido este testigo y utiliza el evento con el mismo fin último, demostrar poder, conseguir deslumbrar y emocionar al público que congregan.
\end{abstract}

Palabras clave: Eventos; comunicación; publicidad; historia.

\section{The events of yesterday and today: a show of power}

\begin{abstract}
:
The event today is a kind of communication where the ultimate goal is the emotional connection between the company and the public. This is an overall experience where everything communicates, It have the power to communicate through feelings, they are spaces where information lives, feel and touch. Therefore, one can derive that the events are tools used efficiently and creatively, have power and they are a demonstration of power .

This technique of communication is not new. If we turn to history, we find the demonstration of power and wealth were the events and who made them were kings. Today, it is the company that has collected this intention and uses the event with the same ultimate goal, demonstrate power, get dazzle and thrill audiences who flock.
\end{abstract}

Key words: Events; communication; advertising; history. 


\section{Referencia normalizada:}

Mut Camacho, M. y Bernad Monferrer, E. (2014): El paralelismo entre el evento de ayer y el de hoy: exhibiciones de poder. Historia y Comunicación Social. Vol. 19. Núm. Especial Enero. Págs. 513-524.

Sumario: 1. Introducción. 2. Los valores comunicacionales del evento. 3. El evento en la historia. 4. Estudio de casos: el evento antiguo. 5. Reflexión final. 6. Referencias bibliográficas

\section{Introducción}

En la actualidad los eventos se han convertido en una forma de comunicación muy utilizada por las empresas, pero a lo largo de la historia ha sido el modo comunicativo de las culturas tradicionales cuando se perseguía un fin colectivo.

Hasta hace pocos años tan solo la utilizaban con asiduidad aquellas empresas que tenían vetadas otras formas de hablar sobre su producto, como es el caso de las farmacéuticas o las tabacaleras, pero ya no es así, con frecuencia muchas empresas invierten parte de su presupuesto de comunicación en la realización de eventos, y consecuencia de ello es la proliferación de empresas de comunicación que se dedican en exclusiva, o casi en exclusiva, a la organización de eventos.

Una explicación de este importante desarrollo es que los eventos, tradicionalmente utilizados para comunicar con un público muy limitado pero de alto valor, se han convertido en una eficaz herramienta de comunicación de la marca/empresa, de sus valores y su identidad muy enérgica. Los eventos son auténticas representaciones de las marcas donde convergen varios caminos que no son tan superficiales como podría parecer. Esto es así porque son manifestaciones experienciales de la realidad pero revestida de un halo de emoción que sirve para exteriorizar y explicar a la marca. Es la convergencia de experiencias individuales e ideal colectivo y armónico, y este encuentro puede ser muy fértil en significados para la imagen de marca y para la reputación de la empresa.

Las empresas organizan giras de conciertos, torneos, exhibiciones deportivas, fiestas, reunión de famosos, etc. de forma que, además de servir para emocionar a ese importante público participante del evento a través de una realidad alterada llena de símbolos y de ideales embellecidos, acaba repercutiendo en una imagen global también conmovedora para el que lo observa y para toda la opinión pública a través del altavoz de los medios de comunicación.

El evento de hoy en día es una técnica de comunicación con las virtudes de la variedad, la tecnología y la creatividad, donde el objetivo último es la conexión emocional entre la empresa y el público.

Un evento es una experiencia global donde todo comunica, pensada en función de los intereses de la compañía o la marca, donde la comunicación cara a cara alcanza su máximo exponencial, para que llegue un mensaje determinado a los stakeholders de la organización. Los eventos tienen el poder de comunicar a través de sensaciones: son espacios donde la información se vive, se siente y se toca (Comunicas?, 2007). 
Los eventos siempre se han realizado para deslumbrar y para emocionar, este objetivo de comunicación no es nuevo. En la historia encontramos una época pasada en la que la forma de comunicar poder y fortuna eran los eventos y quien los hacían eran los monarcas. El acto ritual servía para reafirmar, legitimar y publicar la validez del aparato formal de poder (García Bernal, 2006, pág. 71), por tanto, se convertía en una demostración y escenificación de la definición de poder.

Si indagamos en la memoria ya localizamos en las intenciones de buena parte de las actividades comunicativas de los estamentos públicos de épocas pretéritas, sobre todo los monarcas, una clara intención de persuadir y de incidir, a través de las acciones de comunicación, en la conformación de una imagen positiva de la monarquía.

Antes y hoy, el evento se beneficia de la preeminencia social que da a quien lo organiza, del capital simbólico que le otorga, de la exclusividad que emana, del realce que consigue, es un discurso ceremonial y emocional muy eficaz.

Tanto las instituciones de las sociedades tradicionales y las empresas de las sociedades modernas han dominado y deben dominar destrezas y habilidades en códigos de comunicación que acaban siendo rituales de reafirmación de poder, más que actos vinculantes de los sentimientos colectivos, aunque lleguen a ser esto último o se llegue a la reafirmación a través de éstos.

\section{Los valores comunicaciones del evento}

Según la profesora Otero Alvarado los eventos son "actividades o acciones de presencia pública que se producen con carácter singular en la vida de las organizaciones. Hechos corporativos que no forman parte de su desarrollo habitual y que se llevan a cabo de un modo extraordinario y con objetivos muy distintos (Otero Alvarado, 2009)".

En este tipo de comunicación realizada a través del evento, la experiencia de los participantes es fundamental, y en este sentido, su implicación y grado de satisfacción: revertirá positiva o negativamente en el imaginario que el mismo tiene sobre el producto y la marca. Pues una de las funciones principales que tiene la realización de eventos empresariales es que los productos pueden ser recordados más fácilmente por el público objetivo, al generar simpatía en el público por tratarse de un mensaje menos comercial. Además, el evento como instrumento comunicacional es un elemento auxiliar de la publicidad, el marketing y el periodismo ya que la producción de acontecimientos para llamar la atención de los medios de comunicación de masas es una función propia de la publicity con la finalidad de crear noticia.

De esta manera, teniendo como principal objetivo la transmisión de mensajes corporativos/institucionales, a través de un determinado tipo de acciones y un determinado tipo de comunicación relacional, se produce un intento de aproximar los valores de la marca al público a través de una acción altamente experiencial y emocional. 
Los eventos consiguen:

1. La creación o consolidación de valores

2. La transmisión de la identidad del organizador hacia sus públicos y la obtención de reacciones favorables.

3. El posicionamiento del organizador en la mente del público, puesto que el evento funciona como escaparate global.

4. La consecución de un importante impacto emotivo y experiencial. Con todo su esplendor y su correcta y profesional organización, cuidando todos los detalles, se conseguirá construir una muy buena imagen en torno a la institución que lo organiza.

La publicity que acompaña al acto se convierte en un altavoz difícilmente conseguible con otras herramientas de comunicación.

\section{El evento en la historia}

En el pasado la vivencia de un evento se convertía en el mundo de la maravilla que irrumpe en lo cotidiano, de forma que la creación de la satisfacción pública era el triunfo de un poder comunicador que es capaz de sostener las condiciones de un pacto comunicativo desde su propia retórica visual y representativa (García Bernal, 2006, pág. 47), así se convierten en recursos comunicativos que crean complicidad social.

A lo largo de la historia, la conciencia de que la institución monárquica debía hacer esfuerzos por mantener una imagen positiva y conciliadora ha estado presente en quien convenientemente lo supo entender. Así bien, en una carta que el emperador Carlos I de España envía a su hijo Felipe, entre unos consejos para la regencia durante su ausencia sobre justicia, ecuanimidad, templanza y discreción, le aconseja: “ $\ldots y$ aveys de tener horas para ser entre la jente visto y platicado (Kamen, 1997, pág. 125)". El pueblo, la opinión pública, debía tener una buena imagen de la institución monárquica y había que cuidar de que así fuese, con acciones y relaciones que fomentasen esta imagen positiva intencional.

Hoy en día la organización de eventos sirve para comunicar no sólo la identidad de la empresa sino una imagen intencional elaborada a través del carácter particular del evento, convirtiéndose, en cierta forma, en acción comunicativa que construye imagen global. "Los eventos pueden crear una serie de sinergias con las empresas que van desde la traslación de una cierta imagen y caracteres del evento hacia la empresa/ producto, hasta la posibilidad de hacer acciones promocionales con un abanico de posibilidades: inserción de marca en diferentes medios y soportes, creación de stand, intercambios, etc. (García Bernal, 2006, pág. 37)"

En épocas pasadas, la motivación del evento y sus consecuencias no eran muy diferentes a las de hoy en día, se sabía exactamente porqué se hacían los eventos, el 
objetivo de la fiesta pública era preciso, era un instrumento de comunicación para presentarse ante el público, donde se creaba un mundo inventado, un refugio de fantasía y de tiempo festivo. Era la forma que tenían los monarcas de mostrarse ante sus públicos internos y externos. Así pues, los eventos se convertían en espectáculos de poder, actos planificados y pensados al milímetro. Variados eran los motivos por los que poner en marcha la maquinaria del evento: bautizos, bodas, coronaciones, victorias militares, visitas de otros monarcas o embajadores de otros países, entre otros.

Como ejemplo de la importancia y el símbolo de poder que poseía el evento, encontramos al primer candidato a la mano de Isabel la Católica, que nos muestra con su actuación cuán estratégico era el evento. Se trataba del noble D. Pedro Girón, maestre la Orden de Calatrava, con dispensa papal y hermano del Marqués de Villena. Cuando, tras la aceptación real a su propuesta de casamiento, inició el viaje hacia Segovia donde estaba Isabel de Trastámara, llevó consigo tres mil lanzas de guerra y un tesoro de joyas, pedrerías y telas de Holanda. Además le acompañaba en el viaje una larga comitiva formada por toda su corte y los ornatos para la organización de justas, torneos, carreras de cintas, alanceamientos de toros y demás parafernalia que acompañaba una boda de aquella categoría.

Los eventos del poder monárquico llegaron a su apogeo en la época de su biznieto Felipe II y hasta Felipe III. Actos espectaculares en los que se construía arquitectura temporal en la ciudad, cambiando radicalmente su fisonomía, adornándola con arcos de triunfo, escenografías, procesiones, luminarias, fuegos de artificio, desfiles, carros engalanados, torneos, juegos ecuestres, toros, teatro e incluso naumáquias. También se adornaban los puentes y las murallas con estatuas provisionales representando virtudes, seres mitológicos, personajes históricos y seres legendarios. Se disponían fuentes para la ocasión distribuidas por la ciudad, se adornaban balcones y ventanas con tapices y doseles, ramas y flores para el trayecto, lienzos para entoldar calles, decoraciones que imitaban materiales nobles, maderas, mármoles, granitos, jaspes, bronces. Toda la imaginación puesta al servicio de la construcción de la imagen corporativa de la monarquía, con el objetivo de reforzar la idea de poder a través de la sorpresa, la admiración y el asombro. Todo estaba ideado con este objetivo idílico, era como un sueño que desde fuera observan los ciudadanos asombrados ante tanta originalidad, lujo y poder. Esto, no tan solo se conseguía desde el decorado, también desde el protocolo que se utiliza de forma magnífica, el trayecto de la procesión con estricto orden de desfile, el recibimiento por parte de las autoridades, los gestos y actitudes, un acto que dejaba maravillados a los ciudadanos y a los obsequiados.

Toda la ciudad ofrecía una imagen idealizada para recibir o para agasajar al personaje principal, y por asimilación, esa imagen idealizada se transmitía al monarca y a la institución histórica que representaba. 
Ejemplo de naumaquia realizada en Valencia en 1755.

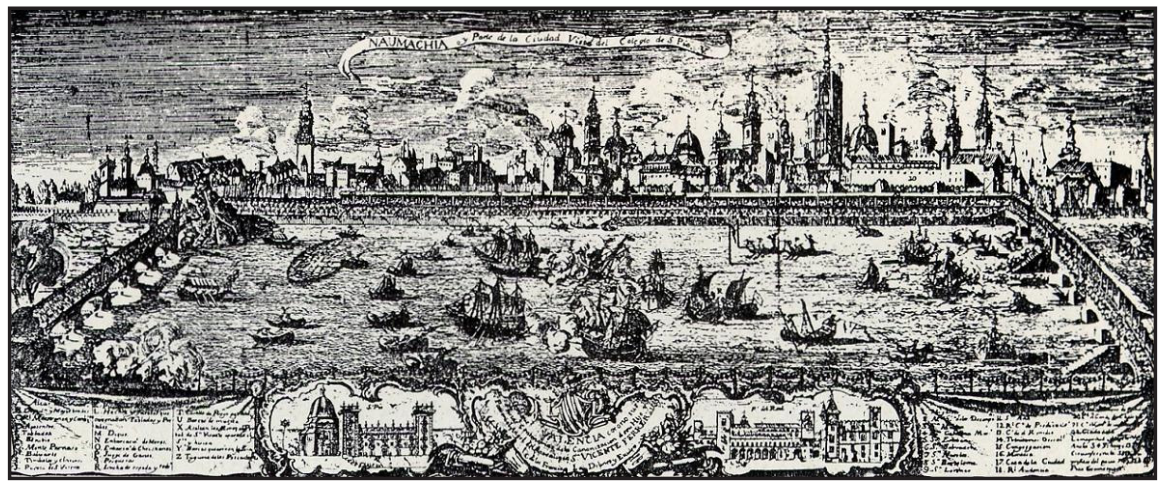

\section{Estudio de casos: el evento antiguo}

En 1534 la ciudad de Salamanca recibió a la primera esposa de Felipe II, la princesa María de Portugal, con unos fastos llenos de una creatividad que sólo se justifica entendiendo la época. En una de las puertas de entrada a la ciudad se alzaba un arco triunfal provisto de varias "nubes", eran unos mecanismos que se abrían y desde él descendían varios niños. Cada uno de ellos iba disfrazado y representaba una virtud en forma alegórica. Uno era el dios romano Mercurio que descendía cantando y recitando versos que explicaban a la nueva princesa que cada una de aquellas nubes se convertía, ante la presencia de los príncipes, en aquellas virtudes de las que la princesa misma era ejemplo. Tras esto había truenos y cohetes, y con música, se abrían el resto de "nubes" y descendían varios muchachos vestidos de doncella representando más virtudes. Con cantos áulicos a los príncipes les hacían entrega de varios objetos emblemáticos de aquello que cada uno representaba: la justicia, una espada; la misericordia, una rama de olivo; las llaves de la ciudad (Ferrer Valls, 1999).

La preparación de estos actos era muy importante para la monarquía y no dejaba nada al azar. Grandes artistas vinculados a la corona como el escultor Pompeyo Leoni o pintores de cámara como Sánchez Coello o Diego de Urbina contribuyeron a la planificación y organización de diferentes eventos que se organizaron durante la monarquía de Felipe II.

Hubo un encargado de eventos ilustrísimo, Don Diego de Velázquez, fue aposentador del rey Felipe IV cumpliendo con su cometido de forma magistral, como cuando en el viaje real que se realizó desde Madrid hasta Fuenterrabía para casar a la infanta, iba por delante de la enorme comitiva, encargándose de acondicionar los lugares por donde pasaba la real comitiva. Se encargó de ir arreglando las estancias con las mejores telas de los palacios reales y adornando los lugares donde se celebrarían los desposorios de la hija del rey, la infanta María Teresa de Austria con su primo hermano el rey Luis XIV de Francia. La boda por poderes se realizó en una iglesia 
de Fuenterrabía transformada, gracias al arte y talento de Velázquez, en una preciosa capilla palaciega.

Los eventos monárquicos además de servir de potenciador de la imagen de poder, servían de propaganda de las gestas de la monarquía y de su vital papel como defensora de la fe católica. Ante todo, era un espectáculo de poder, pues a través de los juegos ecuestres, los señores del reino exhibían sus habilidades a caballo y armados, ante la mirada maravillada del pueblo.

En Salamanca, en 1543, con motivo de la entrada de la princesa María de Portugal, primera esposa de Felipe II, se organizó un torneo en el que se construyó un castillo al final de un vallado guardado por unos gigantes. Los caballeros que se enfrentaban en la contienda hicieron su entrada triunfal acompañados de una serpiente que lanzaba fuego. Del interior de la serpiente salieron doce caballeros para tornear con otros doce que salieron del castillo, a la vez que se lanzaban fuegos artificiales (Ferrer Valls, 1999).

Durante la monarquía de Felipe V, para festejar el bautizo de la última hija del soberano, María Antonia Fernanda, en Sevilla donde estaba la Corte, se celebró un grandísimo evento que duró dos días. El primero por la mañana se lidiaron doce toros; por la tarde treinta y dos caballeros de la Real Maestranza, en briosos corceles andaluces, celebraron una justa y después se mataron siete toros más. El lujo que derrocharon durante todo el evento fue deslumbrante. La creatividad del evento, como de costumbre, fue extravagante: uno de los padrinos de la infanta iba seguido de veinticuatro esclavos negros con grilletes, cadenas y collares de plata. Al día siguiente, por la mañana, hubo lidia de otras doce reses y por la tarde una corrida de rejones en la que tres caballeros, acompañados de cincuenta lacayos, mataron quince toros (Ferrer Valls, 1999).

La estrategia de los eventos también se ponía al servicio de la renovación de alianzas y fidelidades políticas a la monarquía hispana, pues España era un lugar geográfico y político de gran importancia en aquella época. La adhesión de voluntades pasaba por saber utilizar las técnicas de construcción de marca/empresa con maestría, para generar sentimientos positivos. Para ello se buscaba el transmitir una imagen positiva, una imagen de poder, era un propósito esencial en la puesta en escena de la monarquía de cara a sus públicos.

El evento por el bautizo del heredero de Felipe III, Felipe Dominico Víctor de la Cruz, coincidió con la llegada del embajador de Inglaterra, Charles Howard, Conde de Nottingham, que venía a firmar la Paz de Londres y se le quiso deslumbrar.

El "sarao de encantamento" (De los Ríos, 1834, pág. 418), nombre con el que Góngora bautiza este evento en un soneto irónico, fueron diecisiete días de fiestas. Parte de la conmemoración se celebró en un salón del Palacio Real de Valladolid adornado con pinturas de Carducho y la soberbia serie de tapices "La conquista de Túnez", de Vermeyen y Pannemaker, donde se muestra una visión clásica y heroica de las hazañas de Carlos I. Uno de los días de festejo se representó en este salón una 
alegoría en la que la infanta Ana Mauricia, de cinco años, y seis meninas encarnaban a las virtudes; luego hubo un gran baile de gala (Reyes, 2006).

La utilización de los eventos como fórmula para conseguir transmitir una imagen de poder, no era exclusivo de España, los eventos se llevaban a cabo por todo el continente europeo, las casas reales y nobleza de solera desplegaban todo su poderío en los momentos precisos.

La creatividad de los eventos de esta época hoy en día nos puede sorprender mucho, pero eran el reflejo de un tiempo que hoy sólo podemos entender con comprensión y alguna dosis de ternura. El siguiente caso es paradigmático de esta cuestión. Este evento sucedió en París, donde se vivió la fiesta más grandiosa que se había hecho hasta el momento en ese país. Era 1612, hacía dos años que Enrique IV había muerto y el rey francés en ese momento tenía 11 años, Luís XIII. Su madre, la reina regente María de Médicis, lo había prometido con la infanta de España Ana de Austria. Al mismo tiempo, su hija Isabel debía casarse con el heredero Felipe, hijo de Felipe III de España. Era necesario celebrar grandiosamente esta doble alianza de las dos coronas.

La regente, mujer de carácter como muchas Médicis, hizo el evento tan grandioso como grande era la oposición protestante al casamiento, era una cuestión de compensaciones.

En la enorme Place Royale (actual Place des Vosgues en el Marais) se comenzó a preparar el magno espectáculo que tendría como invitado al embajador de España, que había ido a París como representante del rey de España, a pedir la mano de la princesa Isabel. La fiesta duró tres días ininterrumpidos, del 5 al 8 de abril. De este magno evento nos ha quedado un minucioso retrato por la profusión de crónicas, dibujos, estampas y grabados que se hicieron celebrando el acontecimiento.

La regente había encargado a su gran mariscal requisar los balcones para distribuir la corte así como construir un tablado en la parte oeste para los invitados de honor. Los parisinos y el pueblo en general debieron contentarse con los arcos de las galerías y los bordes de la plaza. Los grabados nos muestran multitudes de curiosos que escalaban los tejados. Muchos vinieron los días anteriores para dormir entre los arcos y no perderse la fiesta desde un buen lugar (Perceval, 1995, pág. 46). 


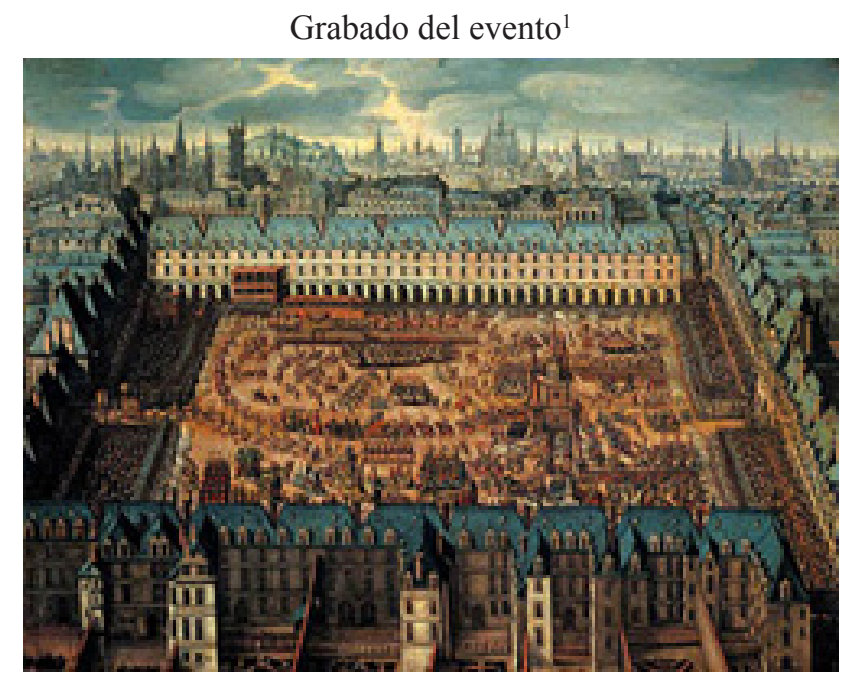

Se escogió como tema de la fiesta uno muy a la moda, las novelas de caballería. El programa del evento constaba de un recorrido por las novelas de caballerías llamado Le roman des Chevaliers de la glorie. François Rosset, el escritor que realizó la traducción del Quijote al francés, dejó un libro donde contaba todo el argumento de este evento sin parangón. La fiesta discurría de la siguiente manera: cinco caballeros, después de múltiples proezas, terminan siendo vencedores de gigantes y dragones, todo ello enmarcado en una recreación de un mundo fantástico y excéntrico.

En una graduación jerárquica, la fiesta era recibida en primer lugar por el pueblo como una incomprensible y fantástica demostración de fuerza y riqueza. Simplemente, contemplaban el despliegue de un fasto casi irreal (Perceval, 1995, pág. 46).

El acto desplegó carros alegóricos a la alianza, hombres y mujeres disfrazados que recitaban versos, cantaban e iban acompañados de bandas de música. Al mismo tiempo se lanzaban cohetes y se montaban castillos de fuegos artificiales. El carrusel importando de Italia constituía una atracción. El pabellón de la reina se había transformado asimismo en un castillo de madera llamado el Palacio de la Felicidad.

La idea era crear un ambiente de cuento de hadas a golpe de timbal y de pólvora, de colorido y de lujoso despliegue de disfraces. Cada día, mientras sonaban los cañones de la Bastilla, la reina ocupaba su lugar en la tribuna real junto a los defensores del castillo, los caballeros de la gloria. Enfrente aparecían treinta caballeros con trompetas y treinta vestidos a la turca, todos con ropas plateadas, que acompañaban a los cinco gigantes con los que lucharían los caballeros. Unos caballos disfrazados de leones llevaban unos carros y otros caballos, disfrazados de unicornios, se enfrentaban a ellos. También desfilaron elefantes y rinocerontes. 


\section{Reflexión final}

No cabe lugar a dudas: los eventos tienen poder y son, en sí mismos, una demostración de poder por promover la participación del público a través de la representación que se prepara con un tono dramático y emocional, consiguiendo trasladar una sensación de dominio y superación.

El evento a lo largo de la historia ha sido un símbolo de poder por la capacidad de organización y de movilización, de originalidad y de derroche. Era el método encargado de construir la imagen de la monarquía a través de actos multitudinarios que despertaban admiración, emociones y sensaciones. Podrían considerarse los eventos como manifestaciones de reafirmación de poder porque se desarrolla una retórica ceremonial que declara grandeza por el despliegue de medios abrumadores que hace que se vea exaltada su superioridad.

Hoy también se construye imagen a través de los eventos, pero ahora el lugar de los reyes y nobles lo han tomado las empresas, creando eventos pensados para conseguir despertar las sensaciones en estado puro y directo, el mismo objetivo que se perseguía antes. En la sociedad tradicional se buscaba que el mundo de la maravilla irrumpiese en lo cotidiano y ahora también, el evento corporativo es la demostración de un mundo estético y lleno de significados para la marca que compromete al sujeto participante y le da protagonismo.

Las empresas persiguen, y consiguen, el mismo impacto al crear eventos exhibiendo un importante elenco de famosos, desplegando un significativo derroche de lujo y posibles, con originales demostraciones de tecnología y creatividad; todo ideado para impactar, para que el evento se convierta en una experiencia única, para conseguir diferenciarse ofreciendo momentos sublimes, sensaciones y experiencias nuevas. El evento, de esta forma, se concibe como una demostración de poder que consigue añadir valor a la marca/empresa que causa admiración y cautiva al espectador.

Su envoltura emocional coincide con la gran puesta en escena de un poder cuya lejanía y superioridad debe 'arriesgarse' públicamente para seguir despertando confianza. Y el riesgo asumido se mide, para empezar, en la capacidad de creación, de desplegar un espectáculo que busca captar y conmover a mil mirades (García Bernal, 2006, pág. 133)

La crisis económica ha traído consigo una fuerte polarización de los eventos en nuestro país. Los recursos económicos de las organizaciones se dirigen sobre todo a promover eventos de influencia externa con un claro foco en promover las ventas, en detrimento de otros eventos como los vinculados a la motivación del público interno.

La crisis ha repercutido en que actualmente se invierta menos dinero en producción, por lo que ya no se encuentran fácilmente grandes puestas en escena, los detalles son austeros, el catering ha virado hacia lo sano, sencillo y sostenible, etc. (Eventoplus, 2013). 
Los elementos que hoy en día se están abriendo camino con gran fuerza son los tecnológicos: redes sociales, la web 2.0, audiovisuales innovadores como el mapping 3D, las Apps y la versatilidad de los iPads y las tablets.

La necesidad de aunar creatividad y austeridad está dando pie a la creación de formatos innovadores como los multiobjetivos, los multistakeholders o la creciente tendencia de los eventos virtuales y especialmente de los híbridos donde los formatos estándares conviven con las redes sociales o con sistemas de geolocalización que permiten la interacción entre participantes y organizador.

Herramientas eficacísimas, que utilizadas con buena estrategia y mucha creatividad, pueden ayudar a situar al evento como una eficaz forma de contactar con el público que desea relacionarse con la marca en un ambiente de sensaciones.

\section{Bibliografía}

BERNAD, E. (2011): "Eventos y ciudad: los eventos como elementos clave para la proyección territorial" Actas Icono14 - II Congreso Internacional Ciudades Creativas - Revista de Comunicación y nuevas tecnologías. Pg. 1709.

COMUNICAS?. (2007). Número 8. Barcelona. Pág. 6.

DE LOS RÍOS, V. (1834): Análisis del Quijote y Vida de Cervantes. Barcelona.

EVENTOPLUS (2013): "Grupo eventoplus presenta su Estudio de mercado 2013: del show al business" Disponible en: http://www.eventoplus.com/articulo/1958/4/ grupo-eventoplus-presenta-su-estudio-de-mercado-2013-del-show-al-business/ (Consultado el 15 de septiembre de 2013).

GARCÍA BERNAL, J. J. (2006): El fasto público en la España de los Austrias. Universidad de Sevilla. Sevilla.

FERRER VALLS, T. (1999): «Las fiestas públicas en la monarquía de Felipe II y Felipe III», en Glorias efimeras: las exequias florentinas por Felipe II y Margarita de Austria, Sociedad Estatal para la Conmemoración de los Centenarios de Felipe II y Carlos V.

FERRER, T. (1999)“Las fiestas públicas en la monarquía de Felipe II y Felipe III”. www.uv.es/entresiglos/teresa/pdfs/fiestaspub.PDF!. (Consultado el 15 de septiembre de 2013).

KAMEN, H. (1997): Felipe de España. Siglo XXI. Madrid.

OTERO ALVARADO, M. T. (2009): Protocolo y organización de eventos. UOC, Barcelona.

PERCEVAL, J.M. (1995): Bodas reales que cambiaron la historia. Planeta. Barcelona.

REYES, L. (2006): “El ceremonial de bautizos de las dinastías españolas está registrado desde 1571, en tiempos de Felipe II". http://www.historiarte.net/articulos/ art018.html (Consultado el 15 de septiembre de 2013). 


\section{Nota}

1 Inauguration de la place Royale par un ballet équestre intitulé « Le roman des chevaliers de la gloire » à l'occasion du mariage de Louis XIII avec Anne d'Autriche huile sur bois, école polonaise, XVIIe siècle. Paris, musée Carnavalet. Recuperado el 20/8/2013 de www.culture.gouv.fr/culture/actualites/ celebrations2005/vosges.htm

\section{La autora}

Magdalena Mut Camacho. Doctora en Comunicación empresarial e institucional por la Universitat Jaume I y licenciada en Ciencias de la información por la Universidad Autónoma de Barcelona. Profesora del Departamento de Ciencias de la Comunicación de la UJI. Es codirectora, junto a Joan Costa, del Máster Internacional de Dirección y Gestión de la Comunicación para Latinoamérica. Su actividad docente e investigadora se centra en la gestión intangible de la organización y en la comunicación corporativa. Ganadora del I Premio Prat Gaballi del Colegio de Publicitarios y RRPP de Cataluña. Dirige el grupo de investigación Reputation, Brand and Innovative Communication Towards Competitiveness. Es miembro fundador del Foro Iberoamericano sobre Estrategias de Comunicación y miembro de la Asociación de Directores de Comunicación DIRCOM. 